\title{
Normal and anomalous behaviour of electric, magnetic and seismoacoustic signals recorded in the Amare cave
}

\author{
Francesco Bella $\left({ }^{1}\right)$, Pier Francesco Biagi $\left({ }^{2}\right)$, Michele Caputo $\left({ }^{3}\right)$, Giuseppe Della Monica $\left({ }^{1}\right)$, \\ Anita Ermini $\left({ }^{4}\right)$, Wolfango Plastino $\left({ }^{1}\right)$ and Vittorio Sgrigna $\left({ }^{1}\right)$ \\ ( $\left.{ }^{1}\right)$ Dipartimento di Fisica, III Università di Roma, Italy \\ (') Dipartimento di Fisica, Università di Bari, Italy \\ (3) Dipartimento di Fisica, Università «La Sapienza», Roma, Italy \\ $\left(^{4}\right)$ Dipartimento di Scienze e Tecnologie Fisiche ed Energetiche, Università «Tor Vergata», Roma, Italy
}

\begin{abstract}
Since 1987 multichannel instrumentation has been recording electromagnetic and seismoacoustic emissions in the Amare cave (Gran Sasso - L'Aquila). Equipment detecting RMC (Principality of Monaco) longwave broadcasting $(216 \mathrm{kHz})$ has been operating in the same place. Data collected during this period have pointed out two different phenomena called «quiet» and «perturbed» that characterize the normal behaviour of the cave. On 25 August 1992 an earthquake with $M=3.9$ occurred in the Gran Sasso area and on 4 June 1993 an earthquake with $M=4.3$ occurred in Umbria, $100 \mathrm{~km}$ to north of the Amare cave. Before these earthquakes, electromagnetic, seismoacoustic, and RMC data showed anomalies. Here we present the observed phenomenology and discuss the possibility that the anomalies can be considered precursors of the earthquakes.
\end{abstract}

Key words electromagnetic signals - earthquake precursors

\section{Introduction}

Multichannel equipment able to record electromagnetic and seismoacoustic emissions has been placed in natural caves at the purpose to investigate earthquake precursory phenomena. In 1987 analog equipment for measuring the electric component was put into operation in the Amare cave located in the Central Apennines (Bella et al., 1987). A digital acquisition system was set up later and new sensors, able to measure the electric and the magnetic component of electromagnetic emissions and seismoacoustic signals, were installed (Bella et al., 1989). In 1991 the equipment was completed setting up one radio antenna to detect Radio Monte Carlo (Principality of Monaco) longwave broadcasting $(f=216 \mathrm{kHz})$. The instrumentation and the main features of the Amare cave are detailed in previous papers (Bella et al., 1994a, 1994b).

\section{Results}

The electric, magnetic and seismoacoustic signals (EME, MAG, ACU) collected in the Amare cave seem to have a behaviour of two types we call «quiet» and «perturbed» (Bella et al., 1994a, 1994b). A quiet state is characterized by the absence of significant seismoacoustic, LF electric, and LF magnetic signals and by continuous signals on the IF and HF electric bands and on the IF magnetic band. A per- 
turbed state is characterized by the sudden appearance of significant seismoacoustic signals in all three ACU bands simultaneously. On these occasions there are also continuous clear electric signals. On the same occasions, magnetic signals very often appear in the LF and IF bands. The RMC signals have a 24-hour modulation characterized by a nightly enlargement, and a drop in the signal is always present between 1 and 5 a.m. (when radio broadcasting ceases). These signals do not differ between the quiet and perturbed states.

From 17 August to 24 August of 1992 a particular phenomenology appeared: seismoacoustic signals not coupled with electric or magnetic signals during a perturbed state (fig. 1). Because this was the only time such a phenomenology appeared, it must be considered an anomaly. For clarity, only some of the signals are shown in fig. 1.

On 7 May 1993 another particular phenomenology, shown in fig. 2a, appeared: an abrupt decrease of RMC signals, lasting about twelve days (such a circumstance had never happened before). At this time there was no significant variation in the other (electric, magnetic, and seismoacoustic) signals. The possibility that the decrease was connected with the RMC broadcasting station was excluded because the RMC signals recorded in the same period in the Cervo cave $(50 \mathrm{~km}$ from the Amare cave) did not decrease (fig. 2b). The phenomenology observed in the Amare cave must therefore be considered an anomaly.
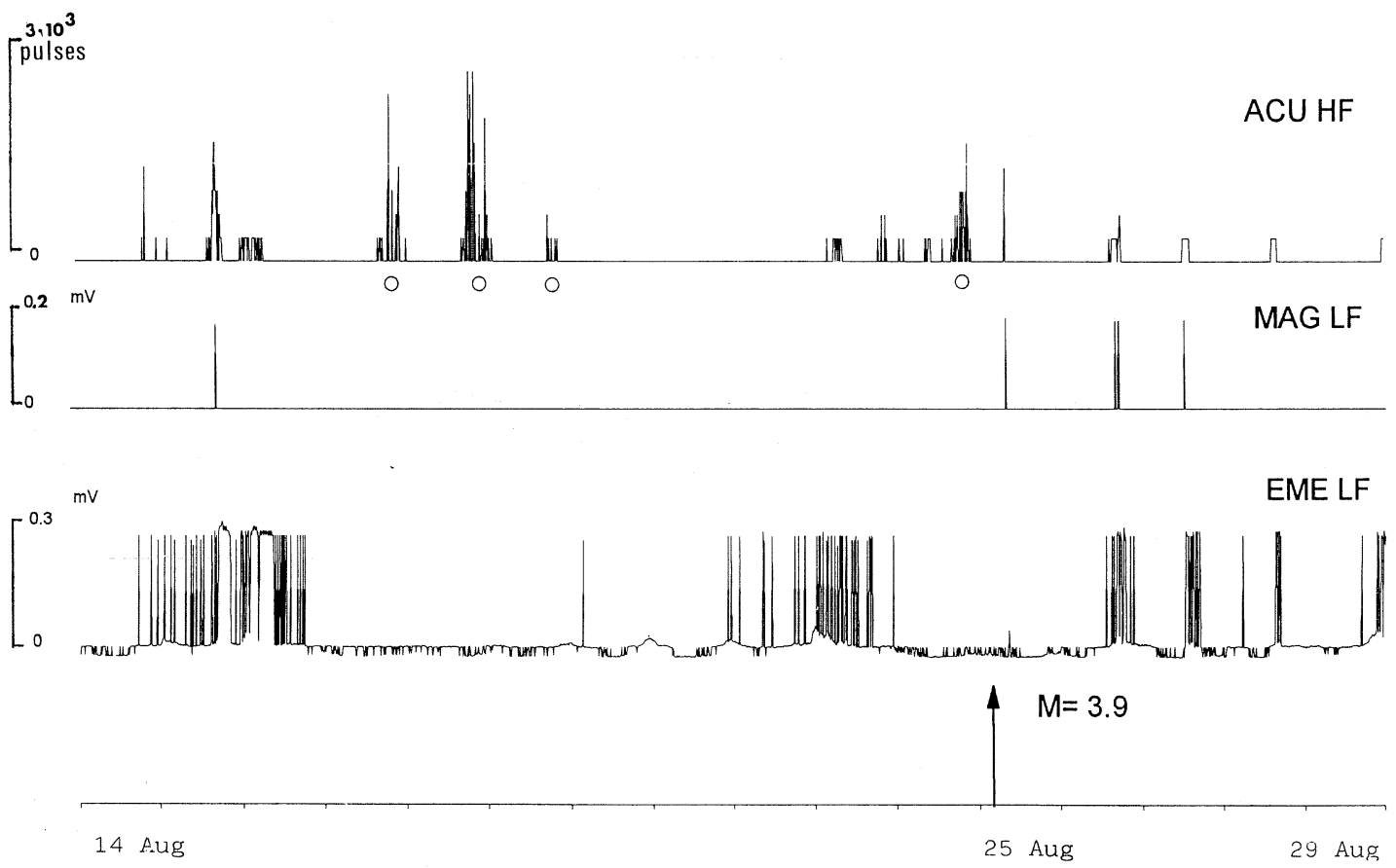

Fig. 1. Electric, magnetic (both as integrated output voltage) and seismoacoustic (as number of pulses) signals recorded from 14 August to 29 August of 1992. The circles mark those days when seismoacoustic and EME and MAG signals were not coupled. The arrow indicates the occurrence of the 25 August earthquake. 


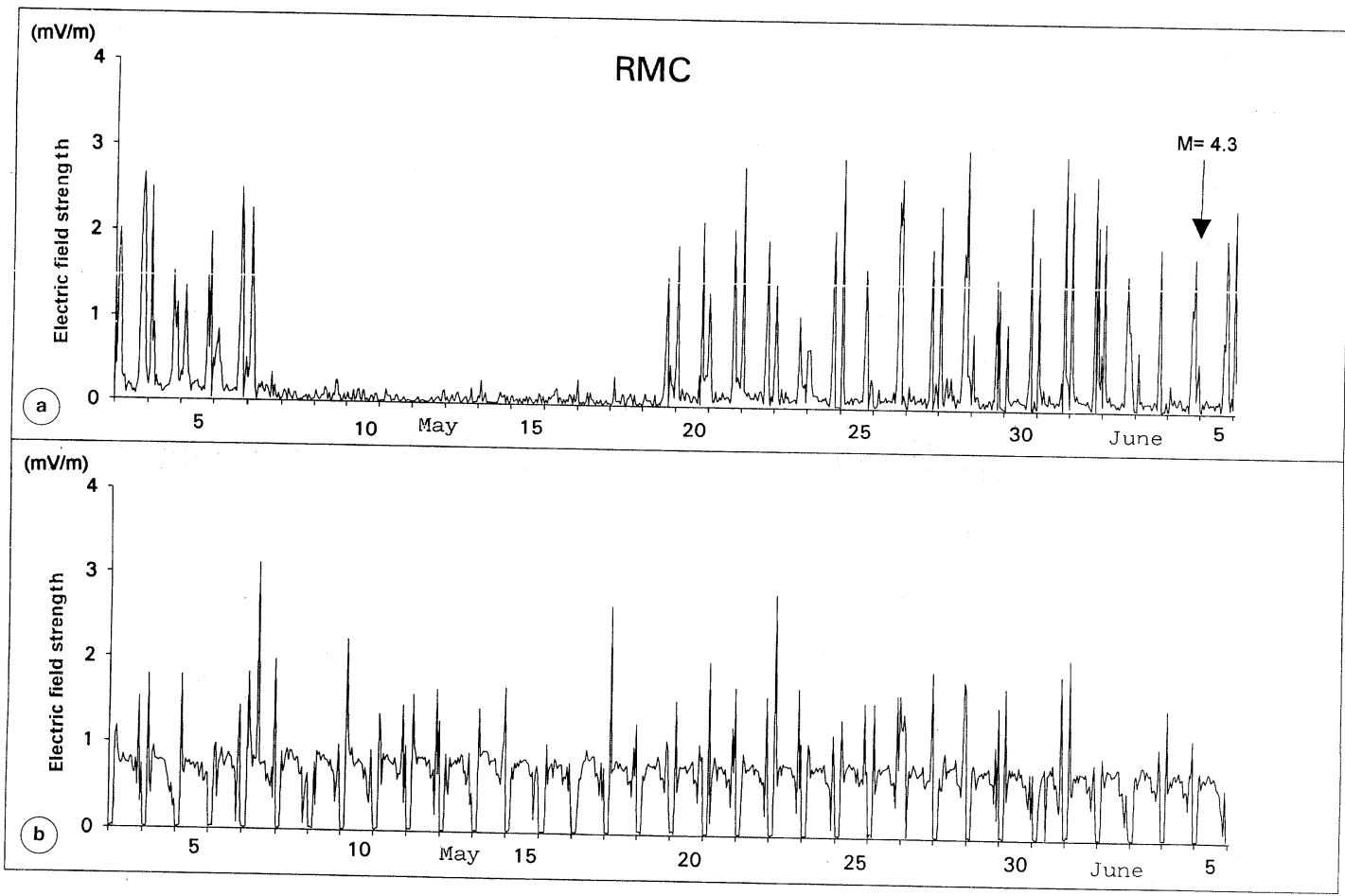

1993

Fig. 2a,b. RMC electric field strength recorded simultaneously: a) in the Amare cave; b) in the Cervo cave.

\section{Discussion}

With regard to the quiet and the perturbed state, the features of both of them and the possible causes (atmospheric pressure variations and rainfall) that can change one situation into another have been discussed in previous papers (Bella et al., 1994a, 1994b).

Now consider the anomalies we described in the previous section. The first thing to be noted about the phenomenology of August 1992 , is that the meteorologic data identify this period as a perturbed one. The anomaly thus consists of both a decrease of electric (and magnetic) signals and the appearance of a new type of seismoacoustic signals. On 25 August 1992 an earthquake with $M=3.9$ occurred less than $20 \mathrm{~km}$ from the cave: its relative deformation parameter $\varepsilon$ (Dobrovolsky et al., 1979) is the largest one $\left[510^{-8}\right]$ obtained during the six years of measurements. The occurrence of the earthquake is shown by an arrow in fig. 1. It seems natural to consider the anomalous phenomenology we have described as a precursor of this event. The uncoupling of ACU signals from EME (and MAG) signals indicates that the ACU signals have different origins from those characteristic of any perturbed state. We propose the following explanation: the uncoupling of the signals is related to the effect of the preseismic stress that increases the cohesion between the blocks, «freezing» the whole system. At the same time, the preseismic stress produces micromovements or microfractures or 
both in the area, near the future epicentre. The seismoacoustic signals recorded in the Amare cave are just those generated in these processes. The remarkable decrease (with respect to the normal conditions) of the absorption coefficient of the elastic waves in stressed rocks could result in the path of seismoacoustic signals being considerably longer (Armstrong, 1969). The lack of such anomalous phenomenology on the occasion of events with lower $(<3.9)$ magnitude which happened in the same area is justified by a stored energy too low for generating preseismic effects.

Now consider the phenomenology we observed in May 1993. We can exclude the possibility that this phenomenology is connected with some variations in the geomagnetic indexes, both because of the consistent duration of the phenomenon and because the signals recorded in the Cervo cave do not show any variation. On 4 June 1993 an earthquake with $M=4.3$ took place about $100 \mathrm{~km}$ north-northeast of the Amare cave. It must be noted that this event is the strongest one to have occurred since 1991 within a radius of $100 \mathrm{~km}$ from the measurement site. The occurrence of this earthquake is shown by an arrow in fig. 2a. We think that the anomalous situation we reported is a precursor of the earthquake. A possible interpretation is as follows: the RMC signal as a terrestrial wave goes through the focal zone of the forthcoming earthquake, and the enhanced stress in this zone during the preparation phase of the earthquake modifies the characteristics of the subionospheric channel in which the RMC signal propagates. This signal might thus undergo more absorption and diffraction processes, decreasing the signal intensity. The fact that such a decrease is revealed by the instrumentation set up in the Amare cave and not by that installed in the Cervo cave $50 \mathrm{~km}$ east (fig. 2a,b) can be interpreted by taking into consideration the different paths that the RMC signal takes from the broadcasting station to the two measurement sites.

\section{Conclusions}

The results presented here contribute to our knowledge of the electromagnetic precursory phenomena of earthquakes. It is important to note that any anomalous situation would be undetectable if the background were not defined completely.

\section{REFERENCES}

Armstrong, B.H. (1969): Acoustic emission prior to rockbursts and earthquakes, Bull. Seismol. Soc. Am., 59, 1259-1279.

Bella, F., P.F. Biagi, G. Della Monica, A. Ermini, P. Manjgaladze, V. SgRigna and D. Zilpimiani (1987): Underground monitoring system of electromagnetic emissions, Il Nuovo Cimento, 10C, 495-504.

Bella, F., R. Bella, P.F. Biagi, G. Della Monica, A. ERmini, P. Manjgaladze, V. Sgrigna and D. ZILPIMIANI (1989): A digital recording system of electromagnetic emissions, Il Nuovo Cimento, 12C, 251-259.

Bella, F., R. Bella, P.F. Biagi, M. Caputo, G. Della Monica, A. Ermini, W. Plastino and V. Sgrigna (1994a): Artificial and natural electro-magnetic signals revealed during two years in the Amare cave (Central Italy), Annali di Geofisica, 37 (5 suppl.), 1131-1136.

Bella, F., P.F. Biagi, M. Caputo, G. Della Monica, A. ERmini, W. Plastino and V. SGrigna (1994b): Electromagnetic background and preseismic anomalies recorded in the Amare cave (Central Italy), in Electromagnetic Phenomena Related to Earthquake Prediction, edited by M. HAYAKAWA and Y. FUJINAWA (TERRA Pub. Comp., Tokyo), 181-192.

DOBROVOLSKY, I.P., S.I. ZUBKOV and V.I. MiACHKIN (1979): Estimation of the size of earthquake preparation zone, PAGEOPH, 117, 1025-1044. 